Ferner wurden den ballistischen Messungen noch Werte der Remanenz, der Permeabilität im Remanenzpunkt, der reversiblen Magnetisierungsarbeit und der Sättigungsmagnetisierung entnommen, über die später berichtet werden soll.

Eléktronenmikroskopische Untersuchungen der Nickelschichten ergaben, daß das Nickel orientiert auf dem Kupfer aufwächst. Die Kristallite besitzen eine mittlere Ausdehnung von 0,5 $\mu$ innerhalb der Schichtebene. Das Dimensionsverhältnis der Kristallite innerhalb der Schichtebene beträgt im Mittel 1 : 3. Vermutlich bilden diese lamellenförmigen Kristallite auch die Grenzen der Eindomänenbereiche, womit man erklären kann, daß in diesen Schichten Gestaltsanisotropie vorliegt. Die Größe der Koerzitivkraft läßt sich dagegen nach (1) mit Hilfe der Kristallenergie berechnen, wenn die Kristallite in allen Richtungen etwa die gleiche Ausdehnung besitzen.
Experimentell wurde dies mit elektrolytischen Nickelschichten auf einer auf Glas aufgedampften Kupferschicht erreicht. Die mittlere Kristallitgröße betrug bei diesen Schichten 300 Å. Es ergibt sich ein ganz anderer Verlauf der Koerzitivkraft in Abhängigkeit von der Schichtdicke. Die Koerzitivkraft fällt unterhalb $1 \mu$ auf etwa 40 Oe ab. Die Temperaturabhängigkeit der Koerzitivkraft ergibt in diesem Falle keine Proportionalität mit $J_{\mathrm{s}}$, sondern sie ergibt, daß in diesem Falle die Kristallenergie wirksam ist, da diese bei $-190^{\circ}$ um einen Faktor 5 größer ist als bei Zimmertemperatur.

Zwischen diesen beiden Extremfällen kann man je nach der Kristallitgröße alle möglichen Übergänge finden ${ }^{4}$. Über diese Untersuchungen wird zu einem späteren Zeitpunkt ausführlich berichtet werden.

${ }^{4}$ L. R e im e r, Vortrag auf der Physikertagung in Wiesbaden (1955).

\section{Erzeugung zeitlich aufgelöster Spektren von Hochfrequenz-Funkenentladungen kleiner Energie}

\author{
Von Á. B a rdó c z u. F. Varsán y i
}

Ungarische Akademie der Wissenschaften, ZentralforschungsInstitut für Physik, Spektroskopische Abteilung, Budapest, Ungarn

(Z. Naturforschg. 10 a, 1031-1032 [1955]; eingegangen am 23. Mai 1955)

Die Aufgabe, zeitlich aufgelöste Spektren von Hochfrequenz-Funkenentladungen relativ kleiner Energie aufzunehmen, ist dadurch erschwert, daß die Lichtausbeute einer einzigen solchen Funkenentladung zur Schwärzung der photographischen Platte nicht genügt. Es muß vielmehr der Lichteffekt von mehreren Hundert oder Tausend Funkenentladungen verwendet werden, um eine brauchbare Aufnahme des Spektrums zu erhalten. Da zur Untersuchung von zeitlich aufgelösten Spektren eine Auflösung in der Größenordnung von Mikrosekunden oder noch darunter nötig ist, müssen die mehrere Hundert oder Tausend zeitlich aufgelösten Spektren genau aufeinander photographiert werden. Unsere Arbeit gibt die Beschreibung einer Vorrichtung, welche zur Herstellung solcher zeitlich aufgelösten Spektren geeignet ist.

Bekanntlich können zeitlich aufgelöste Spektren mit Drehspiegeln hergestellt werden. Das Bild der Lichtquelle wird parallel ${ }^{1,2}$ oder senkrecht ${ }^{3}$ über den Spalt des Spektrographen hinweg geführt.

Da im ersteren Fall das zeitliche Auflösungsvermögen diejenige Zeit ist, in der sich das Bild der Lichtquelle um seine eigene Länge weiter bewegt, ist das Auflösungsvermögen um so größer, je kleiner das Bild der Lichtquelle in der Längsrichtung der Spaltes, je größer die Laufgeschwindigkeit des Bildes längs des Spaltes

1 G. G o r d o n u. W. M. C a d y, J. Opt. Soc. Amer. 40, 852 [1950].

2 L. B lit z e r u. W. M. C a d y, J. Opt. Soc. Amer. 41, 440 [1951).

3 F. T s u i, Brit. J. Appl. Phys. 3, 139 [1952]. und je kleiner die zeitliche Streuung des Eintretens der Funkenentladung ist. Während nun das Bild der Lichtquelle prinzipiell nach Belieben verkleinert und die Laufgeschwindigkeit des Bildes beliebig gesteigert werden kann, ist die zeitliche Streuung des Eintretens der Funkenentladung von den Eigenschaften des Funkenerzeugers abhängig. Bei den bisher verwendeten spektroskopischen Funkenerzeugern war die zeitliche Streuung so groß ${ }^{4}$, daß die zeitliche Auflösung von Funkenentladungen mit Megahertz-Frequenzen nicht in Betracht kam. Das von B a r d ó c z eingeführte FunkenerzeugerSystem ${ }^{5,6,7}$ ermöglicht eine bessere zeitliche Auflösung als eine Mikrosekunde. Bei diesem Funkenerzeugersystem wird die Funkenentladung durch ein auf das Gitter einer Thyratronröhre gegebenes Zeichen ausgelöst. Die Funkenentladung setzt im Verhältnis zu diesem Zeichen mit einer Zeitstreuung von einigen Zehntel Mikrosekunden ein. Dadurch wird die Genauigkeit des Einsetzens der gebräuchlichen Funkenerzeuger um etwa zwei Größenordnungen verbessert.

Die zur Herstellung von zeitlich aufgelösten Spektren benützte Apparatur ist aus der Abb. 1 ersichtlich. Die Abbildung zeigt unten das Schaltschema des elektronisch gesteuerten Präzisions-Funkenerzeugers. Der Kondensator C wird über die Schaltelemente RI-T-V1 aufgeladen, wo T ein Hochspannungs-Transformator mit dem Übersetzungsverhältnis 220/15 000 und V1 eine Gleichrichterdiode ist. Der Kondensator wird über die Funkenstrecken A und S entladen und liefert hiermit die Anregungsenergie. Die Thyratronröhre V2, deren Gitter von der Photozelle I über den Verstärker K gesteuert wird, leitet die Entladungen ein. Die Wirkungsweise des Funkenerzeugers ist in den Arbeiten ${ }^{5,6,7}$ beschrieben.

Abb. 1 zeigt oben die Anordnung des optischen Systems. Das von der Funkenstrecke A kommende Licht fällt durch den Spalt B auf den konkaven Drehspiegel L,

\footnotetext{
4 H. K a i s e r, Z. techn. Phys. 19, 399 [1938].

5 Á. B a r d ó c z, Nature, Lond. 171, 1156 [1953].

6 Á. B a r d ó c z, Spectrochim. Acta (im Erscheinen).

7 Á. B a r d ó c z, Acta Phys. Hungarica 4, 91 [1954].
} 
der auf der Achse des Synchronmotors D befestigt ist. Die Elektroden und der Spalt E der Funkenstrecke A liegen senkrecht zur Zeichenebene, der Spalt B dazu parallel. Eine Seite des Spiegels ist mit schwarzem Papier belegt. Der Spiegel L bildet den Spalt B auf dem Spalt E des Spektrographen verkleinert ab.

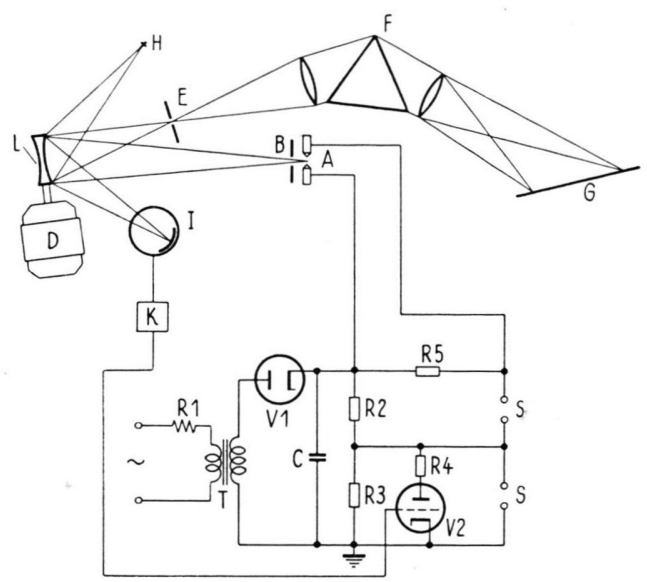

Abb. 1. Vorrichtung zur Aufnahme von zeitlich aufgelösten Spektren. Der untere Teil stellt das elektrische Schaltschema des elektronisch gesteuerten Funkenerzeugers, der obere die optische Anordnung dar.

* Abb. 2 und 3 auf Tafel S. 956 b.
Die Steuerung der Röhre V2 zwecks Einleitung der Funkenentladung erfolgt dadurch, daß der Glühfaden der Autolampe $\mathrm{H}$ durch den Spiegel L vergrößert auf den in der Zeichenebene liegenden Spalt der Photozelle I abgebildet wird. Der bei jeder Umdrehung des Motors ausgelöste Photostrom gelangt über den Verstärker K als Zeichen zum Gitter der Thyratronröhre V2. Die negative Vorspannung erhält die Röhre V2 ebenfalls vom Verstärker K.

Abb. $2 *$ und 3 zeigen gemäß Abb. 1 photographierte Spektren von Magnesium und Antimon. Beide Aufnahmen wurden bei $3 \mathrm{~mm}$ Funkenstrecke mit dachförmigen Elektroden gemacht. Die Kante der Dachform lag parallel zur optischen Achse. Bei den dargestellten Aufnahmen betrug die Kapazität des Kondensators C 10000 pF, die Schwingungszahl des Entladekreises ungefähr 0,5 MHz. Die Weite des Spaltes B war 0,45 mm, die Umdrehungszahl des Drehspiegels 3000/sec. Der Krümmungshalbmesser des Spiegels L war $360 \mathrm{~mm}$. Der Spalt B wurde auf dem Spalt E dreifach verkleinert abgebildet. $1 \mathrm{~mm}$ Länge der Spektrallinie auf der photographischen Platte $\mathrm{C}$ entsprach annähernd 5 Mikrosekunden. Als Zeitmaß ist die zeitliche Intensitätsschwankung der Spektrallinien der Abb. 3 gut brauchbar. Die Periodizität der Intensitätsschwankung beträgt 2,14 Mikrosekunden, identisch mit der Periode des Funkenerzeugers. In den Aufnahmen ist die zeitliche Auflösung etwa 1 Mikrosekunde. Für eine Aufnahme waren ca. 500 Funken nötig.

\section{Bemerkung zu einem von $H$. Welker gefundenen „Transistor-Effekt" in Indium-Phosphid}

\author{
Von D. A. J e n n y
}

Radio Corporation of America, RCA Laboratories, Princeton, - New Jersey, USA

(Z. Naturforschg. 10 a, 1032-1033 [1955] ; eingeg. am 22. Oktober 1955)

In der Arbeit „Über neue halbleitende Verbindungen II" beschreibt W elk e r ${ }^{1}$ kurz einen Spitzentransistor-Versuch mit p-leitendem InP. Die Beeinflussung der Kollektorkennlinie durch Modulation des Emitters wird mit Hilfe eines Oszillographenbildes veranschaulicht und als „Transistor-Effekt" gedeutet. Im Verlaufe unserer Arbeiten auf dem Gebiete der halbleitenden Verbindungen hatten wir Gelegenheit, diesen „Transistor-Effekt“ etwas genauer zu untersuchen. Unsere Resultate und die von Welker abweichenden Schlußfolgerungen sollen im folgenden kurz beschrieben werden.

Ein gewöhnlicher Triodentransistor kann im elektrotechnischen Sinne ganz allgemein als ein Vierpolnetzwerk mit wenigstens einem aktiven Element neben den passiven angesehen werden. Der aktive Teil ist notwendig, um Leistungsverstärkung hervorzurufen. Obwohl Leistungsverstärkung eine hinreichende Bedingung für die Existenz eines Transistor-Effektes ist, ist sie keineswegs notwendig. Im physikalischen Sinne ist ein

${ }^{1}$ H. W el k e r, Z. Naturforschg. 8a, 248 [1953].
Transistor-Effekt vorhanden, wenn die folgenden zwei Prozesse nachgewiesen werden können: 1. Emission von Minoritätsladungsträgern in die Halbleiterbasis durch den Emitter und 2. Einfang von wenigstens einem Teil dieser Minoritätsträger durch den Kollektor. Die gleichzeitige Existenz beider Prozesse ist eine notwendige und zugleich hinreichende Bedingung für einen TransistorEffekt, auch wenn keine Leistungsverstärkung nachweisbar ist.

Abb. 1 zeigt eine Schaltung von zwei getrennten(!) Halbleiter-Gleichrichtern mit einem Widerstand und zwei Wechselstrom-Generatoren, wobei der eine einen wesentlichen Innenwiderstand $R_{\mathrm{i}}$ besitzt. Der Meßwiderstand $R_{0}$ ist klein verglichen mit allen anderen Widerständen und dient lediglich der Übertragung des Stromes $i_{\mathrm{c}}$ auf den Oszillographen. Die Generatorfrequenzen sind $f_{\mathrm{e}}=1000 \mathrm{~Hz}$ und $f_{\mathrm{c}}=60 \mathrm{~Hz}$.

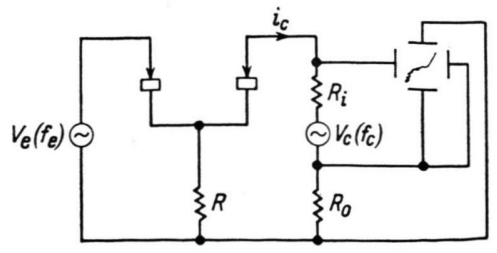

Abb. 1.

Abb. 2 gibt das mit dieser Schaltung erhaltene Oszillographenbild wieder, welches im wesentlichen mit demjenigen Welkers übereinstimmt. Die Tatsache, daß die 